\title{
CIRSE Standards of Practice on Analgesia and Sedation for Interventional Radiology in Adults
}

\begin{abstract}
This CIRSE Standards of Practice document provides best practices for the safe administration of procedural sedation and analgesia for interventional radiology procedures in adults. The document is aimed at health professionals involved in the provision of sedation and analgesia during interventional radiology procedures. The document has been developed by a writing group consisting of physicians with internationally recognised expertise in interventional radiology, and analgesia and sedation.
\end{abstract}

\section{Introduction}

Even if correctly performed, procedural sedation and analgesia (PSA) may increase the risk of morbidity and mortality during the therapeutic intervention. The aim of this document is to provide best practices for the safe administration of PSA [1] to adult patients undergoing interventional radiology (IR) procedures.

PSA can be provided by anaesthetists and non-anaesthesia sedation teams [2] who have acquired the mandatory skills to avoid or safely manage, if necessary, potentially life-threatening adverse events associated with PSA. Non-anaesthesia sedation teams are comprised of personnel specifically trained in PSA, which may include interventional radiologists, registrars in anaesthesia and critical care or radiology, and nurses. Non-anaesthesia professionals have been increasingly involved in the administration of procedural sedation [3-5]. Multiple factors, such as the increased co-morbidities of patients referred to IR, cost-containment, increasing numbers and complexity of IR procedures along with higher expectations among patients, have greatly increased the demand for sedation facilities. With the shortage of anaesthesiologists, scientific societies [4-7] have promoted the use of rapid-acting analgesic and/or hypnotic-sedative drugs by fully-trained nonanaesthesiologists to prevent and manage potentially life-threatening adverse events. The administration of safe PSA requires specific provider competencies which will be addressed in this document.

This document is based on three key papers published in Europe [3], the USA [8], and the UK [9]. The European Society of Anaesthesiology (ESA) and the European Board of Anaesthesiology (EBA) created a taskforce together with European experts in PSA, and developed dedicated guidelines on the topic of PSA in adults [3]. Additionally, the American Society of Anesthesiologists (ASA) has published its own guidance for moderate PSA [8], and the the Royal College of Radiologists relased a document on sedation, analgesia and anaesthesia in the radiology department [9]. These 
important guidelines have been taken into full consideration for the production of the present document by all the writing group members.

In some countries, such as the USA, dedicated bodies, i.e. anaesthesia departments, have to monitor and evaluate PSA according to the Joint Commission on Accreditation of Healthcare Organizations $[3,10]$. Anaesthetists are not required to be directly responsible for sedation services or their quality assurance, but rather to have an advisory and supportive role [10].

As general principles, the choice of sedation technique performed will depend on several factors, including type and duration of the procedure itself, anaesthesiologists' availability, patient's health status, and patients' preference. All these aspects have to be taken into consideration when planning patients' care. PSA can range from local or regional, to general anaesthesia. In addition, percutaneous imaged guided nerve blockade techniques can influence PSA.

\section{Definition}

Sedation is a progressive drug-induced condition whereby a drop in consciousness is induced to allow the patient to tolerate an uncomfortable and/or painful interventional procedure. The process is a continuum from light sedation to deep general anaesthesia. In the present document, the American Society of Anaesthesiologists definition and classification will be used [8].

Four progressive levels of sedation have been classified (Table 1). At levels 3 (deep sedation/analgesia) and 4 (general anaesthesia) the patient is at higher risk of life-threatening complications. For these latter sedation levels, advanced airway management and cardiac monitoring is essential and must be delivered by a trained anaesthesiologist.

\section{Pre-procedural patient assessment and planning}

Although the most appropriate level of sedation should be planned prior to the procedure, unexpected deeper levels of sedation may occur and protocols should be readily available in the IR room to guide the management of potential complications including:

- Immediate rescue of the patient and correction of physiological parameters;

- Anaesthetic equipment availability, as well as very rapid referral to an anaesthetist.

As mentioned in the ASA guidelines [8], anaesthetists must be involved in cases of sedation deeper than mild to moderate and with patients considered ASA physical status higher than II. Specifically, for high-risk patients (ASA physical status III and IV), PSA can only be delivered by an anaesthetist. 
Accurate evaluation and documentation of patients before the IR procedure is important to limit the risk of adverse events. Ideally, patients should be assessed within 30 days of the intended procedure, with a further re-assessment 24 hours beforehand. See Table 2 and Table 3 (ASA physical status classification) for patient assessment.

Standard laboratory tests (adapted to local policies) should be performed. Vital parameters, including heart rate $(\mathrm{HR})$, peripheral saturation $\left(\mathrm{SpO}_{2}\right)$, respiratory rate $(\mathrm{RR})$, and arterial blood pressure $(\mathrm{BP})$ should be documented. Fasting information should be given to the patient as well as to ward staff, as appropriate and according to the most recent ASA recommendations (Appendix 1) [11] .

Medication for chronic illnesses should not be discontinued until the morning of the procedure, with the exception of anticoagulants and antiplatelets [12, 13]. Diabetic patients should follow dedicated peri-operative guidelines according to local policies.

Contact details of the radiology and anaesthesiology services should be given to the patient during the consultation.

Surgical and anaesthetic history should be noted, highlighting any event of difficult airway management (e.g. in previous standard surgical anaesthesia) or any previous history of postoperative nausea or vomiting (PONV) [3]. If considered useful or necessary, pre-assessment by an appropriate specialist (e.g. anaesthesiologist, cardiologist, pneumologist/chest physician) should be considered. The day before or the day of the procedure, the patients should be reassessed.

The specifically dedicated CIRSE IR Safety Checklist [14], or alternatively the World Health Organization Surgical Safety Checklist [15] (even if elaborated for surgical procedures), should be used as a safety procedure.

The following categories of patients may require a specific evaluation by an anaesthesiologist [3]:

- Patients with severe cardiovascular diseases (the Lee index or 'revised cardiac risk' index could be used for this purpose).

- Patients with documented or suspected risk of obstructive sleep apnoea syndrome (OSAS)

- Patients with morbid obesity (BMI $\left.>40 \mathrm{~kg} / \mathrm{m}^{2}\right)$

- Patients with chronic renal failure (glomerular filtration rate $<60 \mathrm{ml} \mathrm{min} / 1.73 \mathrm{~m}^{2}$ for more than 3 months or stage $3 \mathrm{~A}$ )

- Patients with chronic hepatic disease (Model for End-stage Liver Disease score, MELD >10)

- Elderly patients (> 70 years)

- Patients with ASA physical status III to IV 
- Patients with moderate-severe chronic obstructive pulmonary disease (COPD)

- Asthmatic patients

- Patients with neuromuscular or neurological disease (including non-cooperative patients)

- Multi-allergic patients

- Haemodynamically unstable patients (including those who are bleeding).

\section{Monitoring, patient care and equipment}

Patient positioning is very important to prevent pressure and positional injuries, allow safe monitoring, as well as to reduce the need for patient movement due to prolonged procedures in uncomfortable positions. Appropriate peripheral vascular access should be placed and maintained in position until discharge. A $20 \mathrm{G}$ canula is usually sufficient to provide safe sedation. Different access approaches must be individualised depending on the patient and procedure.

Appropriate monitoring, depending on the global clinical context (e.g. comorbidities, procedure, sedation level), should be undertaken prior to the procedure and continued in an appropriate recovery area, to quickly identify the first signs of deterioration of any vital parameter so as to avoid sedation-related complications (e.g. hypotension, arrhythmia, hypoxaemia, hypercapnia).

The procedural sedation and monitoring of the patient during the procedure should be delivered by a member of the procedural team, other than the specialist performing the procedure. In other words, the person who delivers the PSA must be exclusively dedicated to this during the procedure and cannot be simultaneously involved in the IR activity. In addition to standard instrument monitoring (see below), the level of consciousness should also be assessed. This should be done about every 5 minutes, or more frequently according to the clinical context, using verbal responses such as feedback to verbal (or tactile [light tap]) stimulation, to maintain the desired level of sedation and avoiding an unexpected drop in the conscious state. When verbal response is not possible, one can check the patient's ability to give a "thumbs up" or other indication of consciousness.

In order to deliver only minimal to moderate sedation, the following basic monitoring equipment should be available for routine care or to support critical scenarios:

- Pulse oximetry $\left(\mathrm{SpO}_{2}\right)$ - mandatory $\rightarrow \mathrm{SpO}_{2}$ allows monitoring of patients' oxygenation status with a delay between the oxygen blood reduction and a decrease in $\mathrm{SpO}_{2}$ that depends on the device used. Supplemental oxygen should be administered unless contraindicated (e.g. oxygen nasal cannula 2-6 I/min or oxygen mask $0.28-0.6 \mathrm{FiO}_{2}$ or oxygen mask with reservoir for $\mathrm{FiO}_{2}>0.6$ if needed).

- Non-invasive blood pressure (NIBP) - arterial pressure monitoring with 5-minute intervals (or less if considered useful) is mandatory. A baseline before the beginning of the procedure is 
necessary as well. Invasive (intra-arterial) blood pressure monitoring should be applied only in selected cases.

- Electrocardiography (ECG) with a 3-leads modality is mandatory

- Non-invasive capnography monitor - suggested, but not mandatory $\rightarrow$ monitoring the carbon dioxide $\left(\mathrm{CO}_{2}\right)$ is a rapid method to assess patients' ventilation, in terms of respiratory rate and/or airway obstruction and, indirectly, cardiac output. Clinical signs of ventilation should always be assessed.

Processed electroencephalography ( $p E E G$ ) is not recommended during light and moderate sedation. In case of anaesthetist-performed deeper sedation procedures, pEEG could be of benefit [16].

Alarms on vital parameters must be checked and properly set, in order to quickly identify changes in a patient's condition.

An electronic or non-electronic medical record must be used to register timing, drugs (including fluids) with attention to including doses and route of administration, vital parameters, and setting (monitoring, patient position).

Basic and advanced airway equipment should be available with a full range of sizes: source of compressed $\mathrm{O}_{2}$, vacuum device for suction with dedicated catheters, face masks and ambu-bag, oropharyngeal cannulae, lubricant,supraglottic airway devices, laryngoscopes, endotracheal tubes, stylets, cricothyroidotomy kit should all be available. A dedicated trolley that includes all the material for difficult airway management should be rapidly available.

A resuscitation cart with battery-powered defibrillator and pacemaker should be promptly available. For respiratory and cardio-vascular support, the following medications should be promptly available: antagonists (flumazenil and naloxone) and emergency medication (epinephrine, ephedrine, norepinephrine, atropine, nitroglycerin, amiodarone, lidocaine, glucose, chlorpheniramine, hydrocortisone, methylprednisolone, dexamethasone, benzodiazepines, adenosine, beta-blockers, magnesium sulphate).

\section{Common analgesic and sedative agents}

Analgesia and sedation are closely interconnected and are delivered using different combinations of drugs that include hypnotics, opioids and non-opioid analgesics (Table 4).

- A pharmacologic strategy should be planned before the procedure starts. 
- Minimising anxiety and pain, which are strongly related, is the target of drug administration. Even if many medications are available as oral preparation (e.g. benzodiazepines), an intravenous (i.v.) administration route due to predictable bio-availability is preferred, but oral drugs may be used in specific situations (e.g. claustrophobia for diagnostic exams).

o Age is a predisposing factor for higher sensitivity and prolonged effect of both benzodiazepines and opioids, especially when combined.

- Each drug (e.g. hypnotic and analgesic) should be administered individually in a step-by-step fashion.

- Administer i.v. drugs in small, progressive additional doses, or by continuous infusion, titrating to the desired endpoints based on the procedure, and the age, weight, co-morbidities and medical history of the patient. Respect the onset time of each drug before readministering additional doses. Timing for absorption and peak effect must be taken into account when drugs are administered by non-i.v. routes (e.g. oral, rectal, intramuscular, transmucosal).

\section{Reversal agents}

When safe sedation is performed, it is unusual to need reversal agents against medications such as sedatives (BDZ) and opioids (Table 5). Nevertheless, a protocol for correct use of reversal agents should be available where PSA is performed.

Table 6 and 7 summarise the drug pharmacokinetics including doses, elimination half times, contraindications and safety issues.

\section{After the procedure: recovery and discharge}

After the procedure, the patient should be transferred to an appropriate recovery area and monitored by dedicated personnel (e.g. IR nurse). ECG, $\mathrm{BP} \mathrm{SpO}$, sedation level, respiratory rate, occurrence of nausea and vomiting, and pain level should be monitored and recorded.

The time of discharge must be recorded and the patients can be discharged to the destination area (ward, home) when [6, 8]:

- The consciousness level has returned to the pre-procedural state.

- The vital signs are stable or returned to the pre-procedural levels.

- An adult responsible for the patient is present when the patient is transferred home.

- Pain is adequately treated.

- Nausea or vomiting are not present.

- The patient has understood and received the post-procedural instructions, including medications and contact details in case of an emergency or questions on aftercare. 
- A chart summarising the procedure performed with post-procedural instructions has been provided to the medical and nursing staff of the receiving ward.

- The patient has been monitored in an appropriate recovery area for at least 30 min after PSA.

- Additionally, a score (e.g. Aldrede's scoring system) could be applied according to local policies. For instance, the Aldrete's scoring system is a used scale for determining when patients can be safely discharged from the post-anaesthesia care unit to the postsurgical ward [17].

\section{Complications}

PSA may result in a wide range of complications during or even after the end of the procedure. Complications may occur despite appropriate delivery of PSA, and these range from mild events to life-threatening conditions that need early recognition and rapid management by clinical staff. Many of these latter complications can be managed by the sedation team. Flow-charts should be readily available/on hand to guide the management of potential complications.

The most frequent complications include:

- Agitation: it can be paradoxical or due to pain, and sometimes it can be worsened by further sedative administration.I It may therefore be appropriate to withhold further sedation and to promptly involve an anaesthetist, when needed.

- Hypotension: it can develop from absolute or relative hypovolemia (blood loss, sepsis or anaphylaxis), or may be due to sedation that is too deep. The team should be ready to administer rapid i.v. fluid boluses and/or vasopressors and to call for help from other relevant specialists.

- Nausea and/or vomiting: priority should be given to maintaining a patent airway, and suction devices should always be ready for use. Nausea and/or vomiting may be due to opioids, sudden hypotension or to co-morbidities. Anti-emetics should be given to relieve symptoms.

- Respiratory depression/airway obstruction: this is commonly due to deep sedation. Appropriate monitoring equipment should be available for prompt identification of signs of respiratory depression/airway obstruction (see the section dedicated to the monitoring devices). Basic airway manoeuvres such as chin lift, jaw thrust, suction, and/or oropharyngeal cannula placement may be required to protect the airways, and early anaesthetic involvement may be required.

Personnel and certification for the acquisition and maintenance of minimum technical skills for non-anaesthesia operators.

In the USA, PSA can be provided by personnel authorised by the ASA, which has created a training course that allows the providers to deliver only mild to moderate sedation to ASA physical status 
I and II patients. In this view, for high-risk patients (ASA physical status III and IV), PSA can only be delivered by an anaesthetist and the present recommendations follow the same principles.

A general recommendation is that the operator who delivers sedation should not be employed in any other activity (e.g. performing the IR procedure).

Appropriately trained personnel and credentialled teams should be able to safely deliver mild (anxiolysis) to moderate PSA only. Levels of sedation deeper than moderate must be performed by dedicated anaesthetists, as vital functions and parameters (cardiovascular and/or respiratory) are likely to be depressed and need support.

The training of personnel dedicated to PSA should follow a training process established by a nominated committee within the institution where PSA is performed.

The team dedicated to PSA must:

- Know the indications, objectives and risks of PSA.

- Know and administer analgesics and sedatives.

- Appropriately monitor (clinical and monitored) vital signs both without and under the effect of PSA.

- Recognise early signs of vital parameter deterioration.

- Be able to promptly rescue and correct cardiovascular and respiratory impairment due to any cause (procedure and/or analgesia-sedation).

- Know the drugs for PSA and antagonist (reversal agents) medications including doses, effects, side effects, consequences of synergism due to concurrent administration of other drugs (e.g. benzodiazepines or propofol plus opioids).

- Clearly and promptly understand if supplemental help (e.g. anaesthesiologist) is needed

- Communicate any concern to the team.

- Be able to activate a rapid response team for emergency support within a shared institutional protocol (see below).

It is mandatory that personnel dedicated to the administration of PSA are trained in:

The management of vascular access and airway assistance (e.g. mask ventilation and oxygenation).

- Basic life support and defibrillation (BLSD) and should be advanced life support (ALS) certified (including retraining), unless sedation is provided by an anaesthetist.

Due to the potentially high complexity of modern IR procedures, if an anaesthetist is in charge of managing patients for IR procedures, he/she should ideally have a special interest in this area to develop his/her knowledge and expertise. 
Completion of a specific dedicated training course must be certified by a dedicated authority (e.g. scientific anaesthesiology societies or hospital anaesthesia committees) that may be identified by the public health institution depending on local policies (specific to each country). The dedicated authority could certify the competence of the trainee to provide PSA and allow different privileges according to the standard achieved during the final evaluation.

\section{Summary and conclusions}

We have been witnessing a progressive increase in therapeutic interventions requiring PSA, and non-anaesthesiologist personnel have become more involved in performing PSA within the IR environment. To ensure that PSA is delivered safely, it is vital that the PSA team undergoes specific training in drug administration, patient monitoring and airway management, as well as cardiovascular system support. The anaesthesia team should be available to coordinate and supervise the PSA team activities and training, to ensure the highest levels of safety. When deeper levels of sedation are required, i.e. level 3-4, anaesthetists must be involved, ideally those with a specific interest in IR.

\section{References}

1. Green SM, Krauss B (2002) Procedural sedation terminology: Moving beyond "conscious sedation." Ann Emerg Med 39:433-435. doi: 10.1067/mem.2002.122770

2. Hatzidakis AA, Charonitakis E, Athanasiou A, et al (2003) Sedations and Analgesia in Patients Undergoing Percutaneous Transhepatic Biliary Drainage. Clinical Radiology 58: 121-127 doi: 10.1053/crad.2002.1128

3. Hinkelbein J, Lamperti M, Akeson J, et al (2018) European Society of Anaesthesiology and European Board of Anaesthesiology guidelines for procedural sedation and analgesia in adults. Eur J Anaesthesiol 35:6-24. doi: 10.1097/EJA.0000000000000683

4. Dumonceau JM, Riphaus A, Schreiber F, et al (2015). Endoscopy. Non-anesthesiologist administration of propofol for gastrointestinal endoscopy: European Society of Gastrointestinal Endoscopy, European Society of Gastroenterology and Endoscopy Nurses and Associates Guideline--Updated June 2015. 47:1175-89. doi: 10.1055/s0034-1393414.

5. Gouda B, Gouda G, Borle A, et al (2017). Safety of non-anesthesia provider administered propofol sedation in non-advanced gastrointestinal endoscopic procedures: A meta-analysis. Saudi J Gastroenterol. 23:133-143. doi:10.4103/sjg.SJG_501_16.

6. Sury M, Bullock I, Rabar S, Demott K (2011) Sedation for diagnostic and therapeutic 
procedures in children and young people : summary of NICE guidance. Bmj 342:45-48. doi: 10.1136/bmj.c6819

7. Vargo JJ, Cohen LB, Rex DK, Kwo PY (2009) Position statement: Nonanesthesiologist administration of propofol for GI endoscopy. Gastrointest Endosc 50:1683-1689. doi: 10.1002/hep.23326

8. ASA (2018) Practice Guidelines for Moderate Procedural Sedation and Analgesia 2018: A Report by the American Society of Anesthesiologists Task Force on Moderate Procedural Sedation and Analgesia, the American Association of Oral and Maxillofacial Surgeons, American. Anesthesiology 128:437-479. doi:

10.1097/ALN.0000000000002043

9. The Royal College of Radiologists (2018) Sedation, analgesia and anaesthesia in the radiology department (second edition).

10. Commission The Joint. 2016. https//www.jointcommission.org/ Stand. jcfaqdetails.aspx?StandardsFaqld=1240\&Programld=46. [Accessed 25 August 2016]

11. ASA (2017) Practice guidelines for preoperative fasting and the use of pharmacologic agents to reduce the risk of pulmonary aspiration: Application to healthy patients undergoing elective procedures: An updated report by the american society of anesthesiologists com. Anesthesiology 126:376-93. doi:

10.1097/ALN.0b013e3181fcbfd9

12. Kriegshauser J, Osborn H, Naidu S, et al (2018) Developing Interventional Radiology Anticoagulation Guidelines: Process and Benefits †. J Clin Med 7:85. doi: 10.3390/jcm7040085

13. Patel IJ, Davidson JC, Nikolic B, et al (2012) Consensus guidelines for periprocedural management of coagulation status and hemostasis risk in percutaneous image-guided interventions. J Vasc Interv Radiol 23:727-736. doi: 10.1016/j.jvir.2012.02.012

14. Lee MJ, Fanelli F, Haage $P$, et al (2012) Patient safety in Interventional radiology: A CIRSE IR Checklist. Cardiovasc Intervent Radiol 35:244-246. doi: 10.1007/s00270-0110289-5

15. World Health Organization (2009) Surgical Safety Checklist https://www.who.int/patientsafety/safesurgery/checklist/en/ . Accessed 22 April 2020

16. Purdon PL, Sampson, A, Pavone KJ et al (2015). Clinical Electroencephalography for Anesthesiologists: Part I: Background and Basic Signatures. Anesthesiology 123:93760. doi: 10.1097/ALN.0000000000000841.

17. Ead H (2006). From Aldrete to PADSS: Reviewing discharge criteria after ambulatory surgery. J Perianesth Nurs; 21: 259-267. doi: 10.1016/j.jopan.2006.05.006] 


\section{Appendix 1. ASA recommendations for fasting before sedation, anaesthesia and procedures}

- Clear liquids may be ingested for up to 2 hours before procedures requiring general anaesthesia, regional anaesthesia, or procedural sedation and analgesia.

o A light meal or non-human milk may be ingested for up to 6 hours before elective procedures requiring general anaesthesia, regional anaesthesia, or procedural sedation and analgesia.

o Additional fasting time (e.g. 8 or more hours) may be needed in cases of patient intake of fried food, fatty food or meat.

o Both the amount and type of food ingested must be taken into account when determining an appropriate fasting period.

o Since non-human milk is similar to solids in gastric emptying time, consider the amount ingested when determining an appropriate fasting period.

o In case of urgent or emergent situations where gastric emptying is not possible, do not delay moderate procedural sedation procedure based on fasting time alone; this condition may require an anaesthetic involvement/referral.

o Refer to the specific guideline for additional details [10]. 
Table 1. American Society of Anesthesiologists - Definition of Sedation and Analgesia [5]

\begin{tabular}{|c|c|c|c|c|}
\hline & $\begin{array}{l}\text { LEVEL } 1 \\
\text { Minimal Sedation } \\
\text { (Anxiolysis) }\end{array}$ & $\begin{array}{l}\text { LEVEL } 2 \\
\text { Moderate } \\
\text { Sedation/Analgesia }\end{array}$ & $\begin{array}{l}\text { LEVEL } 3 \\
\text { Deep } \\
\text { Sedation/Analgesia }\end{array}$ & $\begin{array}{l}\text { LEVEL } 4 \\
\text { General Anaesthesia }\end{array}$ \\
\hline Responsiveness & $\begin{array}{l}\text { Normal response to } \\
\text { verbal stimulation }\end{array}$ & $\begin{array}{l}\text { Purposeful* response to } \\
\text { verbal or tactile } \\
\text { stimulation }\end{array}$ & $\begin{array}{l}\text { Purposeful* response } \\
\text { after repeated or } \\
\text { painful stimulation }\end{array}$ & $\begin{array}{l}\text { Unarousable, even with } \\
\text { painful stimulus }\end{array}$ \\
\hline Airway & Unaffected & No intervention required & $\begin{array}{l}\text { Intervention may be } \\
\text { required }\end{array}$ & $\begin{array}{l}\text { Intervention often } \\
\text { required }\end{array}$ \\
\hline Spontaneous ventilation & Unaffected & Adequate & May be inadequate & Frequently inadequate \\
\hline Cardiovascular function & Unaffected & Usually maintained & Usually maintained & May be impaired \\
\hline
\end{tabular}

\section{Table 2. Patient assessment before an IR procedure}

Informed consent from the patient or his/her advocate based on local policies, including both procedural information and benefits and risks of sedation and analgesia.

Demographic and anthropometric data: age, height and weight.

Medical history with special attention to: $\quad$. Functional reserve (metabolic equivalent task (MET)) [8]

- Clinical signs and specific diseases that may expose the patient to increased risk for sedation (sleep apnoea, chronic obstructive pulmonary disease (COPD), smoking, alcohol abuse)

- Renal and hepatic function

- ASA physical status - Anaesthetic assessment is recommended for patients with ASA 3 or higher (regardless of the type of the procedure), in cases of probable difficult airway management and if it is likely that sedation levels deeper than moderate will be necessary

Investigation for allergies

- List of current medications with special attention to anti-coagulants and anti-platelets dedicated protocols must be followed to weigh thrombotic vs bleeding risk

- Chronic use of opioids, that predicts increased analgesic and sedative requirements; in this case anaesthetic referral is suggested 
Table 3. American Society of Anesthesiologists Classification (ASA Class)

ASA 1 A normal healthy patient. Example: fit, non-obese (BMI under 30), a non-smoking patient with good exercise tolerance.

ASA 2 A patient with a mild systemic disease. Example: patient with no functional limitations and a well-controlled disease (e.g. treated hypertension, obesity with BMI under 35, frequent social drinker or is a cigarette smoker).

ASA 3 A patient with a severe systemic disease that is not life-threatening. Example: patient with some functional limitation as a result of disease (e.g. poorly treated hypertension or diabetes, morbid obesity, chronic renal failure, a bronchospastic disease with intermittent exacerbation, stable angina, implanted pacemaker).

ASA 4 A patient with a severe systemic disease that is a constant threat to life. Example: patient with functional limitation from severe, lifethreatening disease, e.g. unstable angina, poorly controlled COPD, symptomatic CHF, recent (less than three months ago) myocardial infarction or stroke.

ASA 5 A moribund patient who is not expected to survive without the operation. The patient is not expected to survive beyond the next 24 hours without surgery. Examples: ruptured abdominal aortic aneurysm, massive trauma and extensive intracranial haemorrhage with mass effect.

ASA 6 A brain-dead patient whose organs are being removed with the intention of transplanting them into another patient.

The addition of " $E$ " to the ASA physical status (e.g., ASA 2E) denotes an emergency surgical procedure. The ASA defines an emergency as existing "when the delay in treatment of the patient would lead to a significant increase in the threat to life or body part."

Abbreviations: ASA, American Society of Anesthesiologists; BMI, body mass index; COPD, chronic obstructive pulmonary disease; CHF, chronic heart failure.

\section{Table 4. Types of sedatives and analgesics}

\begin{tabular}{|c|c|}
\hline Sedatives & \\
\hline Benzodiazepines (BDZ) & $\begin{array}{l}\text { The most common class of sedatives used for minimal sedation. They are both anxiolytics and } \\
\text { have an amnestic effect } \\
\text { Among BDZ, midazolam is the most used. It has a rapid onset time and a relatively short } \\
\text { elimination half-life. } \\
\text { - Onset } 30 \text { to } 60 \mathrm{~s} \text { and the maximum effect is reached after } 13 \text { min [2]. Duration of action is } \\
\text { longer than propofol ( } 20 \text { to } 80 \text { min) [2]. } \\
\text { - Effects could be prolonged in elderly patients. } \\
\text { - As it has no analgesic properties, it is typically combined with opioids during PSA. }\end{array}$ \\
\hline Propofol $(P)$ & $\begin{array}{l}\text { It remains the most common sedative drug. } \\
\text { It has short onset time (30 to } 60 \mathrm{~s}) \text {, predictable duration of action and short context-sensitive } \\
\text { half-time. }\end{array}$ \\
\hline
\end{tabular}


- It has no analgesic properties. Therefore, it is mostly combined with opioids during PSA resulting in a strong synergistic analgo-sedation.

- Rapid onset time and recovery. It is quite easy to titrate, but the therapeutic range is narrow and respiratory depression and loss of airway reflexes is common.

Ketamine $(\mathrm{K}) \quad$. Its major advantage is the property of being sedative and analgesic while preserving the respiratory drive. It can therefore be used as the sole agent for painful procedures. It causes a dissociative sedation and may increase heart rate and arterial pressure. As a consequence, it should be used cautiously in patients with ischaemic heart disease.

- It has a rapid onset of action (30 to $60 \mathrm{~s}$ ) and a moderate duration of action (10 to $20 \mathrm{~min}$ ).

Dexmedetomidine . Dexmedetomidine may be administered as an alternative to benzodiazepine sedatives on a case-by-case basis.

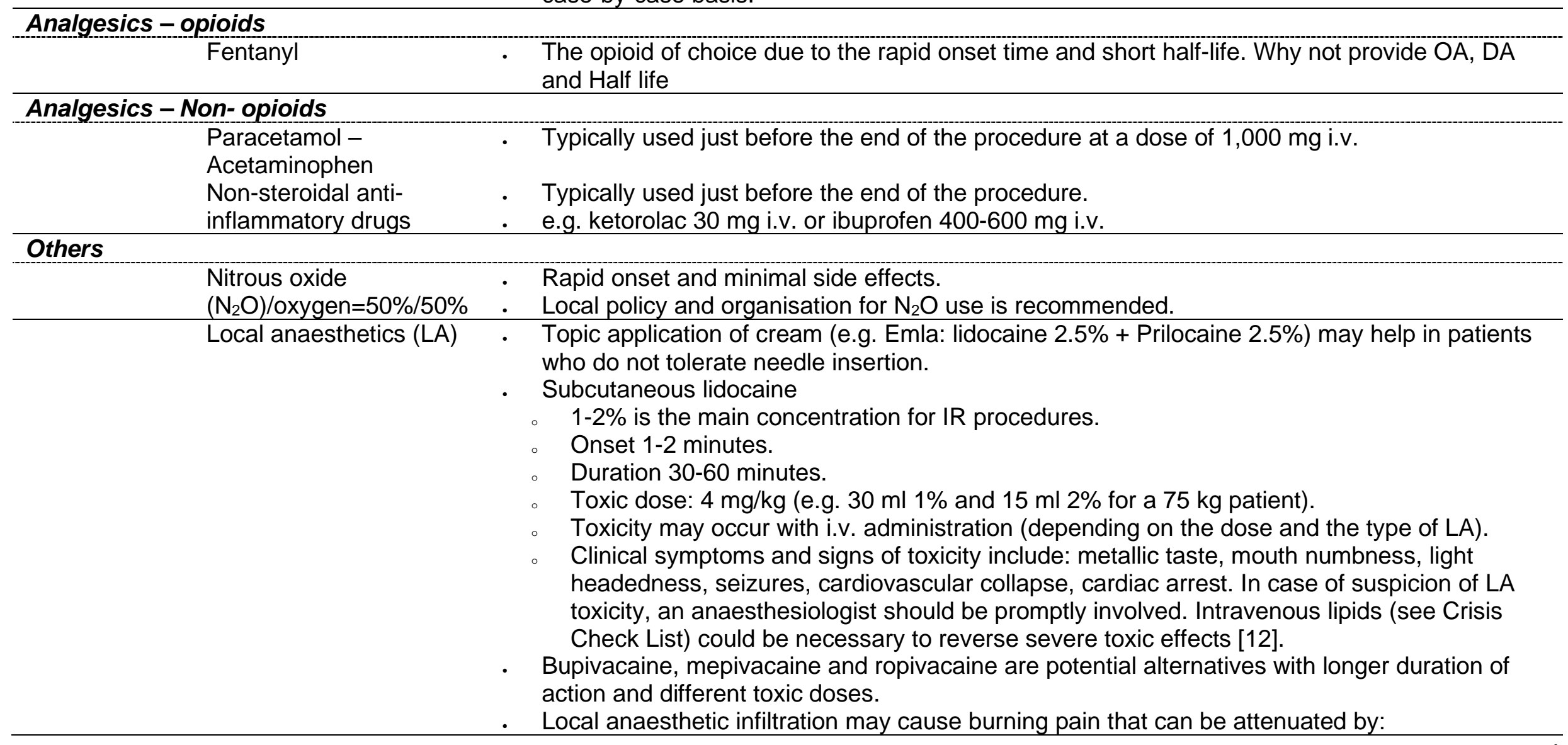




\section{Table 5. Types of antagonist-reversal agents}

\begin{tabular}{lll}
\hline Naloxone & Antagonises the effects of opioids counteracting the respiratory depression (and the analgesic effects). Pain agitation and \\
anxiety are potential side effects. & In order to minimise the risk of side effects, it should be administered in a progressive step-by-step fashion at 0.1-0.2 mg \\
per dose at intervals of $2-3$ minutes until respiratory depression is completely reversed. \\
Its half-life is 90 minutes. Need for re-administration due to opioid effect rebound is uncommon with short-acting opioids \\
like fentanyl. Nonetheless, it is advisable to prolong the observation period in the recovery area.
\end{tabular}

\section{Table 6. Doses of analgo-sedatives}

\begin{tabular}{|c|c|c|c|}
\hline Drug* & Dose (typical) & Pharmacokinetic & Safety issues \\
\hline Midazolam & $1-2.5 \mathrm{mg}$ & $\begin{array}{l}\text { Onset: rapid } \\
\text { Elimination half-life: } 1-4 \text { hours }\end{array}$ & Preparation: $1 \mathrm{mg} / \mathrm{ml}$ \\
\hline Propofol & $0.5-1.5 \mathrm{mg} / \mathrm{kg}$ & $\begin{array}{l}\text { Onset: rapid } \\
\text { Elimination half-life: short }\end{array}$ & Respiratory depression and loss of airway reflexes. \\
\hline Ketamine & $0.1-1 \mathrm{mg} / \mathrm{kg}$ & $\begin{array}{l}\text { Onset: rapid } \\
\text { Elimination half-life: } 2.5-3 \text { hours }\end{array}$ & \\
\hline Fentanyl & $\begin{array}{l}25-100 \text { mcg }(25 \\
\text { mcg steps) }\end{array}$ & $\begin{array}{l}\text { Onset: rapid } \\
\text { Elimination half-life: } 3-7 \text { hours }\end{array}$ & $\begin{array}{l}\text { Stiff chest syndrome is very rare at the } \\
\text { recommended doses }\end{array}$ \\
\hline Morphine & $0.05-0.15 \mathrm{mg} / \mathrm{kg}$ & $\begin{array}{l}\text { Onset: rapid } \\
\text { Elimination half-life: } 2-3 \text { hours }\end{array}$ & \\
\hline
\end{tabular}




\section{Dexmedetomidine}

$0.2-1.4 \mathrm{mcg} / \mathrm{kg} / \mathrm{h}$

\section{Acetaminophen}

$1 \mathrm{~g}$ or $15 \mathrm{mg} / \mathrm{kg}$

Ketorolac

Lidocaine

Ropivacaine

\section{$30 \mathrm{mg}(\max$}

90mg/24h)

Maximum $4 \mathrm{mg} / \mathrm{kg}$

for local infiltration

Maximum 2.5

$\mathrm{mg} / \mathrm{kg}$ in for local

infiltration
Onset: slow in case of administration without bolus induction

Elimination half-life: 2 hours

Onset time: 8 minutes if i.v.

Elimination half-life: $1-4$ hours

Onset: rapid

Elimination half-life: $3.5-9$ hours

Onset time: rapid

Elimination half-life: $0.5-3$ hours

Onset time: intermediate

Elimination half-life: $1.5-7$ hours
Anaesthesiologist use only

Caution: in case of hypotension or bradycardia, severe liver failure

Caution is advised in patients with decompensated liver disease

Caution is advised in frail patients and patients with renal failure; administration no longer than 48 hours Crisis check list readily available in case of local anaesthetic toxicity

Crisis check list readily available in case of local anaesthetic toxicity

*Knowledge of each drug's pharmacokinetic properties is fundamental. Titration of drug to effect is important.

\section{Table 7. Doses of reversal agents}

\begin{tabular}{|c|c|c|c|}
\hline Drug & Dose & Pharmacokinetics & Safety issues \\
\hline Flumazenil & $\begin{array}{l}0.1 \mathrm{mg} / \mathrm{kg} \text { - usually } 0.1-0.2 \\
\mathrm{mg} \text { step boluses }\end{array}$ & $\begin{array}{l}\text { Onset time: } 2 \text { minutes } \\
\text { Half-life: short }\end{array}$ & $\begin{array}{l}\text { Adverse effects: agitation, tremors, seizures } \\
\text { Re-administration may be needed in case of long-acting } \\
\text { BDZ intoxication }\end{array}$ \\
\hline Naloxone & $\begin{array}{l}0.1-0.2 \mathrm{mg} \text { incremental } \\
\text { boluses }\end{array}$ & $\begin{array}{l}\text { Onset time: } 2-3 \text { minutes } \\
\text { Half-life: } 1-2 \text { hours }\end{array}$ & $\begin{array}{l}\text { Adverse effects: pain, agitation. } \\
\text { Re-administration may be needed in case of long-acting } \\
\text { opioid intoxication }\end{array}$ \\
\hline
\end{tabular}

